\section{Xenopus and the art of oxygen maintenance}

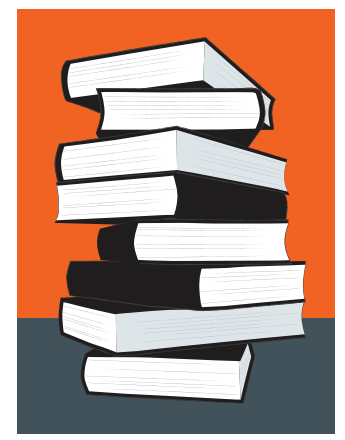

Glenn Tattersall and Warren Burggren discuss the impact of Robert Boutilier and Graham Shelton's classic 1986 JEB paper 'Gas exchange, storage and transport in voluntarily diving Xenopus laevis'.

Robert 'Bob' Boutilier was a deep and philosophical thinker and an experimental wizard. He also had a highly developed sense of humour and irony, considering a pivotal work of his entitled was 'Nautilus and the Art of Metabolic Maintenance' (doubtlessly after Robert Pirsig's cult hit Zen and the Art of Motorcycle Maintenance in 1974). Boutilier's success as a comparative physiologist is chronicled in his many back-to-back JEB articles from the late 1970s through the 1990s, providing seminal contributions on the role of blood gases in breathing and circulation in amphibians and reptiles

(Boutilier et al., 1996). His legacy is also evident in the success of his students (see Ferguson et al., 2004 for a retrospective). We have chosen as a classic article Boutilier's research entitled 'Gas exchange, storage and transport in voluntarily diving Xenopus laevis', co-authored with his PhD mentor, Graham Shelton (Boutilier and Shelton, 1986), and stemming largely from his $\mathrm{PhD}$ studies on $X$. laevis, the African clawed frog (Boutilier, 1981).

The aim of Boutilier's work was to establish '... whether measurements of the rates of depletion and renewal of the lung oxygen store would give some indication of the mechanisms and possible explanations for the extremely variable durations of voluntary dives seen in Xenopus' (Boutilier and Shelton, 1986).
The factors that shape and control intermittent and episodic breathing patterns and the associated cardiovascular adjustments had long been of interest to comparative respiratory physiologists at the time. What was not well understood was how voluntary breath-hold behaviour affected the body's stores for $\mathrm{O}_{2}$ and $\mathrm{CO}_{2}$, how these stores were exploited during intermittent breathing, and how respiratory gases were distributed throughout the body. Considerable description of the phenomenon of intermittent breathing and cardiovascular shunting (often in restrained and/or anaesthetised animals) existed, but their implications to tissue gas exchange were just beginning to be explored. Reflecting Boutilier's technical skills (and patience!), his measurements were conducted in freely behaving unrestrained $X$. laevis (Fig. 1). Previous research had shown that lung blood flow decreases during a dive in X. laevis (Shelton, 1970), but Boutilier proposed three potential functional explanations. They could: (1) minimise cardiac work; (2) regulate movement of $\mathrm{O}_{2}$ from lung to blood as stores in tissues are utilised; and/or (3) drive replenishment of stores while breathing air.

To achieve his goals, Boutilier employed a surgical technique that allowed for continuous recording of lung gases in freely diving frogs. These 'extrapulmonary loops' used a peristaltic pump to draw lung gas and pass it into a gas measurement device for continuous measurement of $\mathrm{O}_{2}$ and $\mathrm{CO}_{2}$, with little disturbance to the frog. Beyond simply measuring partial pressure of $\mathrm{O}_{2}$ and $\mathrm{CO}_{2}$ $\left(P_{\mathrm{O}_{2}}\right.$ and $\left.P_{\mathrm{CO}_{2}}\right)$, Boutilier was meticulous in his recordings and analysis, adopting sophisticated exponential wash-out kinetics in a pre-computer era. Boutilier employed an ingenious mechanical solution to the time delays between electrodes - on his mechanical chart recorder, he simply adjusted one pen to write ahead of the other, thus accounting for the time taken for gas to transit through the tubing between electrodes! Boutilier also implanted indwelling cannulae to measure femoral arterial and pulmonary arterial blood gas partial pressures. Attesting to Boutilier's surgical skills, these cannulae remained patent for days. Boutilier then painstakingly measured lung and blood gases for hundreds of breath-hold periods in $X$. laevis. This combination of clever experimental design, superior surgical skills and innovative analytical approaches enabled Boutilier's unique insights into the gas store usage patterns of intermittently breathing $X$. laevis.

Boutilier first demonstrated that the lungs are the major source of oxygen during diving in $X$. laevis, and that the use of lung oxygen related to dive duration and activity level, stating that although '...a correlation exists between duration of the dive and the rate of lung $P_{\mathrm{O}_{2}}$ decline, the relationships were far from simple and it was never easy to predict when a dive would end...' (Boutilier and Shelton, 1986). One remarkable finding was that voluntary breath-holds differed substantially in oxygen depletion rates (Fig. 2). Not surprisingly, the most rapid depletion rates occurred when animals were spontaneously active, and sometimes depletion rates increased in the latter period of the dive; these breath-hold periods ceased while lung $P_{\mathrm{O}_{2}}$ levels were still quite high. Most striking about the lung gas data was their non-linear naturelung $\mathrm{CO}_{2}$ levels increased to a plateau during a dive, while the $\mathrm{O}_{2}$ levels continually declined. This occurred even with exposure to elevated $\mathrm{CO}_{2}$, where lung $\mathrm{CO}_{2}$ levels simply equilibrated at higher values (Fig. 1). In contrast, lung gas $\mathrm{O}_{2}$ levels generally declined during diving. Exposure to high levels of $\mathrm{CO}_{2}$ in the water tended to lead to shorter dives that ended with relatively elevated $P_{\mathrm{O}_{2}}$ levels in the lungs.

Boutilier implied that factors such as volitional control rather than simply cellular metabolic rate regulated the breath-hold period and lung $\mathrm{O}_{2}$ store depletion rate. This was in an era when the animal behaviour was typically seen as the product of simple reflexes and instinct, and volition in breathing, let alone cognition or personality in animals, was years away from serious consideration. 


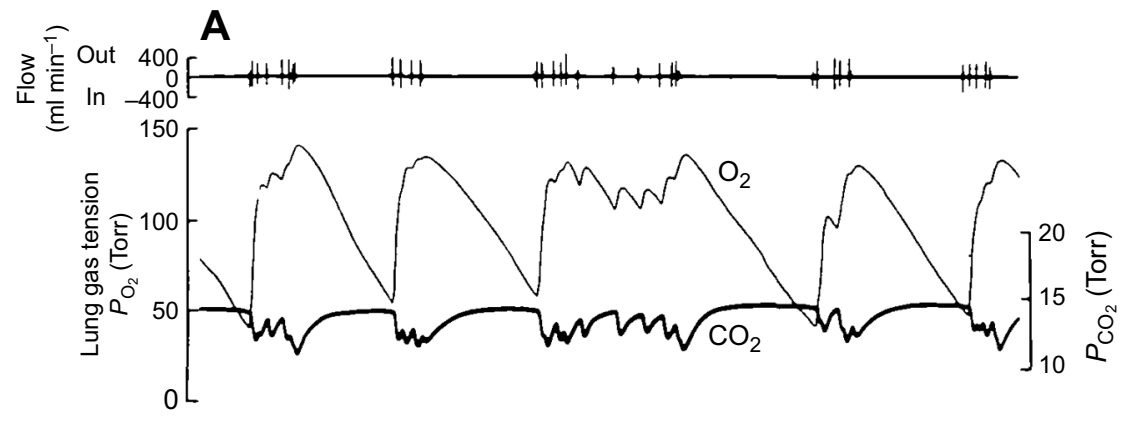

$\underline{10 \mathrm{~min}}$

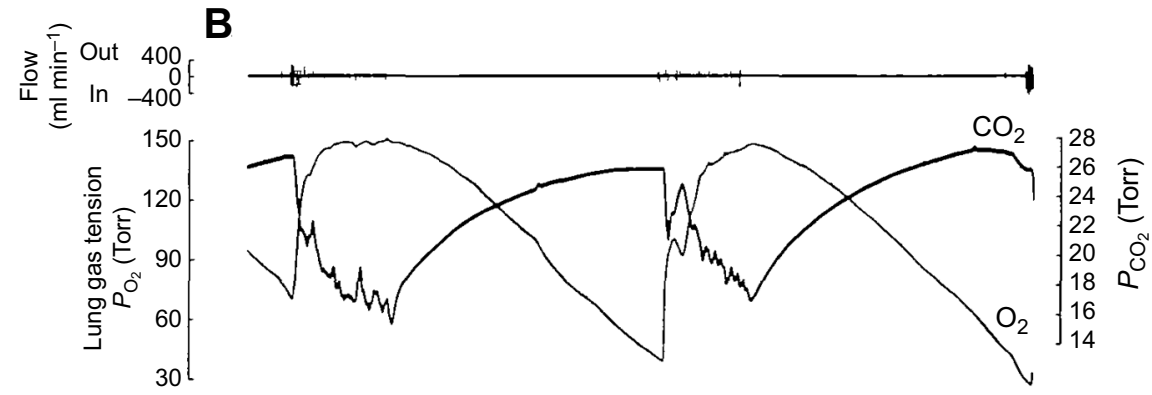

Boutilier's work also contributed vital information to our understanding of vertebrate lung diffusion efficiency, assessed as the gradient between lung and blood partial pressures. This gradient in $X$. laevis was typically $\sim 9 \mathrm{mmHg}$ and changed little throughout diving or throughout ranges of lung fluctuations in oxygen. The $P_{\mathrm{CO}_{2}}$ gradient was also constant, but much lower at $2 \mathrm{mmHg}$. Both gradients were much greater than those observed in mammalian or avian lungs, demonstrating that amphibian lungs have much greater resistance (potentially diffusive or vascular) to gas exchange, reflective of amphibians' lower metabolism. Boutilier provided several reasons for this apparent 'inefficiency', including longer alveolar gas-topulmonary capillary blood diffusion distances, and anatomical shunts between pulmonary artery and vein. He also was among the first comparative physiologists to advocate for inhomogeneities of perfusion in lower vertebrates as the cause of $P_{\mathrm{O}_{2}}$ and $P_{\mathrm{CO}_{2}}$ differences in different lung regions.

Although quantitative data on $P_{\mathrm{O}_{2}}$ values of lung gas, blood and tissues in diving reptiles and amphibians were beginning to accrue through the 1970 s and 1980 s, few researchers were placing these data into predictive models. Not surprisingly,

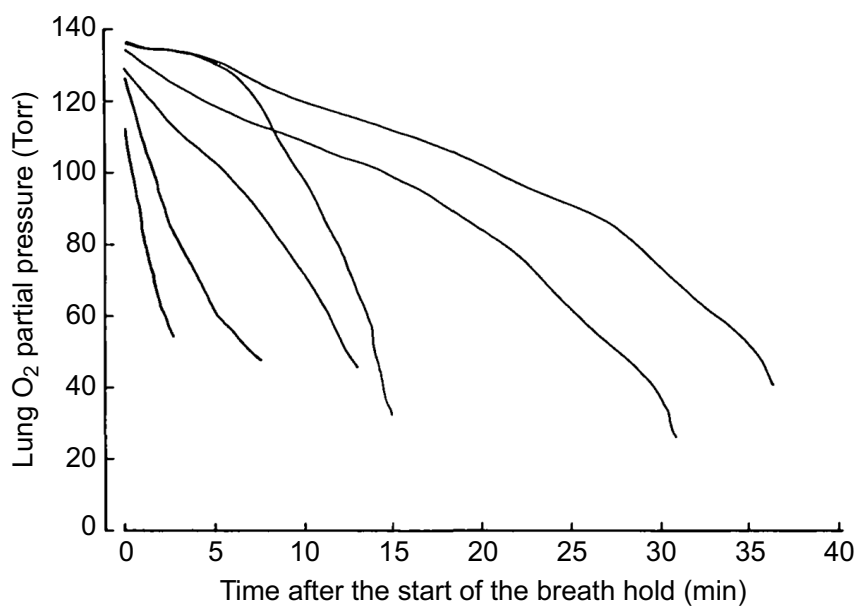

Fig. 2. Boutilier's analysis of variable rates of lung $P_{\mathrm{O}_{2}}$ decline during diving in Xenopus laevis. Not only were these data 'corrected' by accounting for the washout dynamics of the 'extrapulmonary loops' that he designed, but Boutilier went further to evoke volition on the part of these diving amphibians for the first time (from Boutilier and Shelton, 1986).

Fig. 1. Examples of Bob Boutilier's careful investigative methodologies. Sample traces of lung ventilation (depicted as the flow measured by a pneumotachograph) with simultaneous measurements of lung gas tensions in the clawed frog, Xenopus laevis. Noteworthy is that Boutilier conducted these measurements in unanaesthetised, unrestrained frogs that were freely behaving and exhibiting typical diving and surfacing behaviours. In $A$, animals are in air-equilibrated water $\left(25^{\circ} \mathrm{C}\right)$, and in $\mathrm{B}$, animals are in water equilibrated with $1 \% \mathrm{CO}_{2}: 99 \%$ air. Time is indicated by a 10 min line marker (from Boutilier and Shelton, 1986).

Boutilier was innovative on this front. To model the fate of oxygen during diving, Boutilier produced an idealised model, accounting for changes in volume and capacitance of each body store during diving. According to this model, lungs were the primary oxygen store, followed by (especially venous) blood, and finally tissues with the lowest potential oxygen store. Complicating matters was lung volume decrease during diving, which created higher capacitance at high $P_{\mathrm{O}_{2}}$ levels. Turning a complication into an insight, Boutilier concluded that lungs acted more as $\mathrm{O}_{2}$ stores at diving onset when lungs were full. By the end of the dive, venous capacitance for $\mathrm{O}_{2}$ was enhanced, and more critical as an oxygen store at lower $P_{\mathrm{O}_{2}}$ levels occurring later in diving. Boutilier proposed that venous store usage early on in many dives resulted from a classic dive response, whereby reduction in cardiac output resulting from increased systemic resistance led to reduction in systemic blood flow, and increased reliance on venous oxygen supply. The resulting decline in venous oxygen would serve to facilitate oxygen diffusion, by increasing $P_{\mathrm{O}_{2}}$ gradients across the skin, whose role in aquatic gas exchange was being uncovered in the 1980s (Boutilier et al., 1986; Burggren and Feder, 1986; Burggren and Moalli, 1984).

Clear predictions arose from Boutilier's oxygen storage model, especially that changes in blood flow to gas exchange 
organs would determine $\mathrm{O}_{2}$ store depletion rates, which he verified during his post-doctoral research (Boutilier et al., 1986). Vasomotor changes were then predicted by the model to lead to profound changes in store usage. Boutilier proposed that, during diving, increased pulmonary resistance reduced pulmonary blood flow, enhancing the 'right-to-left' shunt, and conserving oxygen stores within lungs and pulmonary vein. Consequently, venous blood stores would then be recruited, augmenting the effectiveness of the cutaneous gas exchanger. In this scenario, lung oxygen stores could then be recruited at the final stages of diving. For active animals maintaining high cardiac output, however, blood flow to both lungs and body remain elevated, which would minimise arterial to venous $P_{\mathrm{O}_{2}}$ differentials; $P_{\mathrm{O}_{2}}$ values in all body stores would converge even closer toward one another. With nearly equivalent $P_{\mathrm{O}_{2}}$ levels, lung $P_{\mathrm{O}_{2}}$ decline would then depend on whole-body $\mathrm{O}_{2}$ capacitance. Thus, lung $P_{\mathrm{O}_{2}}$ would decline quickly in active animals but at ever decreasing rates as the maximum capacitance of body store was reached.

In animals that breathe with only one gas exchange organ (unimodal breathers), partial pressure changes within that organ are simple functions of the total volume of each gas that is exchanged, and in steady state, reflective of metabolic processes. Boutilier highlighted three challenges associated with the vastly more complex picture in bimodal, intermittent breathers. First, the expectation that lung $\mathrm{O}_{2}$ and $\mathrm{CO}_{2}$ levels directly parallel metabolic rate is problematic, as most $\mathrm{CO}_{2}$ is released via the aquatic exchanger, violating assumptions in the classic 'alveolar gas equation'. Indeed, in amphibians, $\mathrm{CO}_{2}$ excretion can be 80 to $100 \%$ accommodated via skin (or gills), rather than lungs (Feder and Burggren, 1985a,b). Second, respiratory steady state is rare in intermittently breathing animals. Consequently, the ratio of $\mathrm{CO}_{2}: \mathrm{O}_{2}$ must change from beginning to end of diving as stores adjust. Third, during diving, assumptions of constant lung volume do not hold, distorting the association between gas partial pressure reflecting actual gas exchange rates. The overall result is that, although at rest and with continuous lung ventilation, $R$-values (defined as the ratio of lung $P_{\mathrm{CO}_{2}}: P_{\mathrm{O}_{2}}$ ) resemble metabolic respiratory quotients ( 0.8), and these values decline during voluntary dives, evidenced by lung $P_{\mathrm{CO}_{2}}$ reaching steady states during diving, while $P_{\mathrm{O}_{2}}$ continues to decline. Therefore, the decreasing lung volume that occurs during a dive is a direct result of the non-uniform replacement of $\mathrm{O}_{2}$ with $\mathrm{CO}_{2}$.

Boutilier exploited the tractability of his model of $X$. laevis bimodal gas exchange to clearly indicate the complexity of this process, which has been appreciated in studies of biomodal breathers ever since.

Boutilier also noted that $X$. laevis has a low scope for change in tidal volume, and thus ventilatory changes are effected through breathing frequency. In an intermittent breather, this occurs by replacing clustering of breathing into bouts of rapid breaths, interspersed by apneic periods. Boutilier's data suggested that declining oxygen levels in lungs and/ or blood may trigger breathing, especially for breathing bouts, although no specific $\mathrm{O}_{2}$ threshold appeared to stimulate breathing. $\mathrm{CO}_{2}$ did not play an important role, either, as lung and arterial levels were low and constant regardless of dive length. Voluntary dives did, however, rarely go below the point of maximum capacitance of body stores. Boutilier concluded that $\mathrm{O}_{2}$ store usage rate was likely of significance in the control of breathing, because dives that ended at high lung $\mathrm{O}_{2}$ levels occurred during high $\mathrm{O}_{2}$ usage rates. His conclusions speak to a classic argument in comparative physiology about the role of oxygen concentration versus partial pressure of oxygen in the sensing of hypoxia (for review, see Milsom and Wang, 2017).

Boutilier and Shelton (1986) ended their now-classic paper with caveats about dive lengths, a question of long interest in the marine mammal literature as well as the respiratory physiology community (see Milsom, 1991; Trassinelli, 2016).

Boutilier concluded that ventilation does not consistently initiate at any particular level of $\mathrm{O}_{2}$ or $\mathrm{CO}_{2}$. Furthermore, following a breath-hold and well after $\mathrm{O}_{2}$ levels had been restored, hyperventilation would continue for some time, suggesting that restoring $\mathrm{CO}_{2}$ stores and acid-base equilibrium is important in deciding when breathing bouts end.

Much of physiology is dominated by a mammalian-centric view centred upon homeostasis and the constancy of the 'milieu interieur'. Yet, intermittently breathing animals, and the morphological and physiological adaptations associated with their respiratory mode, have fascinated comparative physiologists for centuries. From early 19 th century morphological observations through early 20th century physiological experiments, comparative physiologists have strived to understand the physiological and evolutionary implications of intermittent breathing and associated non-steady states. Many incremental advances have been punctuated by a few breakthroughs notable for techniques used, data acquired or analysis that followed. Boutilier's studies of intermittent diving in $X$. laevis exhibited these characteristics, especially in quantitative modelling of oxygen storage dynamics during diving.

As thorough as Boutilier's work was, much remains enigmatic about intermittent breathing, especially the implications of non-steady states for organ systems not directly involved in gas exchange (e.g. kidney, brain). We hope and anticipate that Boutilier's legacy will stimulate a new generation of comparative physiologists to learn from his innovation and insight, and write the next chapters in this ongoing story.

Glenn J. Tattersall Brock University gtattersall@brocku.ca

Warren W. Burggren University of North Texas burggren@unt.edu

\section{References}

Boutilier, R. G. (1981). Gas exchange and transport during intermittent ventilation in the aquatic amphibian, Xenopus laevis. PhD dissertation, University of East Anglia, Norwich, UK.

Boutilier, R. G. and Shelton, G. (1986). Gasexchange, storage and transport in voluntarily diving Xenopus laevis. J. Exp. Biol. 126, 133-155.

Boutilier, R. G., Glass, M. L. and Heisler, N. (1986). The relative distribution of pulmocutaneous blood flow in Rana catesbeiana - effects of pulmonary or cutaneous hypoxia. J. Exp. Biol. 126, 33-39.

Boutilier, R. G., West, T. G., Pogson, G. H., Mesa, K. A., Wells, J. and Wells, M. J. (1996). Nautilus and the art of metabolic maintenance. Nature 382, 534-536.

Burggren, W. W. and Feder, M. E. (1986). Effect of experimental ventilation of the skin on cutaneous gas exchange in the bullfrog. J. Exp. Biol. 121, 445-449.

Burggren, W. and Moalli, R. (1984). 'Active' regulation of cutaneous exchange by capillary recruitment in amphibians: experimental evidence and a revised model for skin respiration. Respir. Physiol. 55, 379-392.

Feder, M. E. and Burggren, W. W. (1985a) Cutaneous gas exchange in vertebrates: design, patterns, control and implications. Biol. Rev. 60, $1-45$

Feder, M. E. and Burggren, W. W. (1985b). Skin breathing in vertebrates. Sci. Am. 253, 126-142. 
Ferguson, R., Iwama, G., Tang, Y., Tufts, B., McAllister-Irwin, N., Claireaux, G., Gamperl, K., von Herbing, L. H., Nelson, J. A., Harper, D. et al. (2004). Thoughts and memories from Bob Boutilier's students and postdoctoral fellows. J. Exp. Biol. 207, 2189-2197.
Milsom, W. K. (1991). Intermittent breathing in vertebrates. Annu. Rev. Physiol. 53, 87-105.

Milsom, W. K. and Wang, T. (2017). Is the hypoxic ventilatory response driven by blood oxygen concentration? J. Exp. Biol. 220, 956-958.
Shelton, G. (1970). The effect of lung ventilation on blood flow to the lungs and body of the amphibian, Xenopus laevis. Resp. Physiol. 9, 183-196.

Trassinelli, M. (2016). Energy cost and optimisation in breath-hold diving. J. Theor Biol. 396, 42-52. 